\title{
A Simple Case of Pallet Setup Features Based Order Picking Routing Optimization
}

\author{
T. Bódis ${ }^{1}$, J. Botzheim ${ }^{2}{ }^{3}$, P. Földesi ${ }^{4}$ \\ ${ }^{1}$ Széchenyi István University, Department of Logistics and Forwarding \\ Egyetem tér 1. 9026, Gyôr, Hungary \\ E-mail: bodis.tamas@sze.hu \\ ${ }^{2}$ Tokyo Metropolitan University, Graduate School of System Design \\ 6-6 Asahigaoka, 191-0065, Hino, Tokyo, Japan \\ ${ }^{3}$ Széchenyi István University, Department of Automation \\ Egyetem tér 1. 9026, Gyốr, Hungary \\ ${ }^{4}$ Széchenyi István University, Department of Logistics and Forwarding \\ Egyetem tér 1. 9026, Győr, Hungary
}

Abstract: Order picking is the most labor-intensive and costly activities in many warehouses by consuming ca. $55 \%$ of the total operating expenses. Order picking development strategies mostly concentrate on warehouse layout, storage assignment policy, routing, zoning and on batching methods. The main challenges of order picking process improvements are the synchronization of these fields and fit the system with the further influencing factors, like unique demands and product parameters. Researchers of the pallet-loading problem could provide a wider horizon on considerable parameters, but their results are rarely implemented into order picking processes. The physical parameters of the products also have a significant impact on the processes which could strongly influence the picking sequence.

The aim of this paper is to describe our own developed methodology for modeling the pallet setup rules. We introduce our product classifying and PSF based decision matrix modeling solutions. These will be the basis of our further research on PSF based harmonized routing and SLA optimization. We examine the complexity of a simple pallet setup features based order picking routing case, where the products of the defined pallet setup classes are stored in separated zones.

Keywords: Order picking; Pallet Loading; Routing; Complexity 


\section{Introduction}

Warehouses have many functions, like receiving, storing in and out, order picking, value added services, final control and forwarding. Order picking has long been identified that, this is the most labor and capital intensive operation. For a typical warehouse, the cost of order picking means 55\% of the total warehouse operating expense. For these reasons order-picking design and management become more important and complex both in manufacturing and distribution [1]. In manufacturing, there is a move to smaller lot sizes, point-of-use delivery, order and product customization, and cycle time reductions. In distribution logistics, in order to serve customers, companies tend to accept late orders while providing rapid and timely delivery within tight time windows. [2]

Designing the order picking system (OPS) is a very complex task which depends on several elements: products, customer orders, different types of functional areas, different combination of equipment types and operating policies for each functional area. [2] [3]

The typical decision problems in design and control of order picking processes (OPP) are the layout design, storage location assignment methods, routing methods, order batching, and zoning [2]. The main influencing factors of order picking time are moving, searching, picking and product stacking time. The primary goal of the OPP development is the routing optimization. According to the scientific literature, traveling time gives the $50 \%$ of the whole picking time. This field is responsible to sequence the items on the order pick list in order to get the shortest route length through the warehouse. The layout optimization takes into account the determination of the number of blocks and the number, length, and width of aisles in the whole storage and picking area [2].

The storage location assignment (SLA) optimization is responsible to allocate products to storage locations for the purpose of lowering routing distance, traveling time, material handling cost and improving space utilization [4]. It let us be able to take into consideration the ordering frequency of items and product parameters, like stacking property. Storage and picking areas can be divided into zones, when the routing optimization has special trade-offs, like progressive and parallel picking which open the question of unit load (UL) consolidating [2]. The Pallet Loading Problem (PLP) requires that the maximum numbers of identical rectangular boxes have to be packed onto a rectangular pallet [5]. However, there are different sizes which have to be packed together. Not to mention, that stock keeping units (SKU) are not only boxes but bags, cans or any amorphous units. Each product has couple of parameters which define their physical stacking property and the required picking sequence (e.g. weight, shape). The mixed SKU property and the specified pallet loading requirements of different partners make the Pallet Loading Problem more complex.

Each influencing parameter of the order picking has impact on the others with different importance which results the complexity of warehousing processes. Many researchers work on the different segments of the OPP development and on the Pallet Loading Problem. 
High number of solutions are defined for harmonizing SLA and routing. For example [6] and [7] optimized the SLA to decrease the routing distances and times. However the researchers usually do not take into account the physical parameters of the products during SLA and routing optimization. Many researchers work on Pallet Loading and Bin Packing Problem, but the solutions are rarely harmonized with SLA and routing algorithms. [8] solved multi-container loading problem and defined order picking sequence, but they did not consider the SLA at the examined warehouse. As we can read it in the study of Bartók and Imreh, there is no algorithm presented for pickup and delivery problem with 3-dimensional loading constraints and with weight limit on the vehicles [9]. Nevertheless, even if new problems will be studied and new models will be developed, still there will exist a gap between practice and academic research [2].

Our research goal is to examine the importance of the Pallet Setup Features (PSF) during warehousing and forwarding processes at different type of warehouses. If the products are sensitive for stacking features, avoiding product damages will have high importance during the picking sequence. Let us introduce it via a simple everyday example. We are going to a supermarket with our shopping list which contains the following items: $2 \mathrm{~kg}$ of potato, 2 dozen of eggs, 2 bottle of soft drink, 2 pack of chips, and 2 box of marzipan figure. We pick all items into our shopping basket. Our goal is to pick items with the shortest lead time, shortest distance, and without product damages. If we pick marzipan figures, chips, and eggs first and put potatoes and soft drinks on the bottom of shopping basket, most of the products will be damaged before paying. So usually we pick the heavy and less sensitive items first (soft drinks, potato) or reconstruct the item sequence in the shopping basket. If the product location assignment supports the right picking sequence we will pick our demands on the shortest way. Otherwise we have to decide, collect items on the right sequence and walk more or pick with shortest routing and redesign the contents of the basket at every item. Both solutions take more time, the best choice depends on the length and content of our list and the product allocation in the supermarket. The mentioned problem is simple, but it well introduces the connections of storage location assignment (SLA), routing, order picking, and pallet setup features in a warehouse [10].

These aspects are not relevant with the same impact at every warehouse which fact opens some questions. Are the physical product parameters relevant at the given warehouse? If those are relevant, what is the rate of importance at a given product group? (i.e. vegetable: high importance, soft drinks: low importance) How can we optimize the order picking with taking into account the pallet setup features? We need to examine the product line, the order characteristics and the warehousing processes to realize the relevance of PSFs at the given warehouses. Usually the companies do not have appropriate data about geometric, weight and stacking parameters of each product and those Stock Keeping Units (SKU). Classifying items based on stacking experiences and defining possible picking sequence could be reliable solution at every warehouse. Modeling and evaluation of the stacking possibilities can identify the importance of stacking aspects. Examination of orders and measuring of warehousing processes are essential, it could highlight many 
movements which make the PSF based OPP development relevant. For example unit load reconstruction time, when the picker has to rebuild the picked unit load to make stable stack. The low traveling speed is also a measurable element, which can be caused by fearing from product damages [11].

The aim of this paper is to describe our own developed methodology for modeling the pallet setup rules and introduce our product classifying and PSF based decision matrix modeling solutions. These will be the basis of our further research on PSF based harmonized routing and SLA optimization. This paper examine the complexity of a simple pallet setup features based order picking routing case, when the products of the defined pallet setup classes are stored in separated zones.

\section{Modeling the pallet setup possibilities}

Pallet Setup Features are unique characteristics of each warehouse. It is important factor mainly at distribution warehouses where order picking has high importance, and the handled products has huge number of variant. The PSF depends on product properties, order parameters and order picking systems.

The possible PSF factors' combination and importance are different warehouse by warehouse which makes the PSF examination and implementation into the order picking algorithms complex. We defined a methodology to model the possible sequencing rules. The resulted PSF based Decision Matrix (PSFDM) let us be able to determine the nature and the complexity of our problem. It defines the pallet setup possibilities and the order picking sequencing rules. It will be the basis of PSF based order picking routing optimization.

The warehouses usually do not have enough and appropriate data about the handled items (i.e. dimensions, shape, weight) or those are changing too often to support a complex PLP or Bin Packing algorithm. However based on known, easily measurable and rarely changing information it is possible to classify the items and every order line. Furthermore the warehouse operator's best practices and experiences are essential and valuable inputs to the classification. The defined Pallet Setup Classes (PSC) have PSF based logical connections (i.e. we should not pick heavy goods up to fragile products) which will be the basis of our PSF based OPP algorithms. Our classification process is described in the following subsections step by step [11].

\subsection{Classification of the product parameter based classes}

We are grouping products based on physical product parameters which has different stacking properties (Eq. (1)). The usually considered factors of Product Classes (PC) are the SKUs, the packaging solution and the item property. Equation (2) shows a possible industrial example [11].

$$
P C=\{A, B, C, D\}
$$




$$
P C=\{\text { PlasticBin,CartonBox,SmallBox,Fragile }\}
$$

\subsection{Classification of the product and order parameter based classes}

We are specifying the defined PCs based on order parameters to define the Product and Order Parameter based Classes (POPC). If it is necessary, we separate PCs into further classes. High quantity of the same item usually has different stacking parameter. For example the element $B$ (CartonBox) of PC set is separated into LowQuantity $(L Q)$ and HighQuantity (HQ) elements (Eq. (3)), (Eq. (4)). In this case if the ordered Quantity $(Q)$ is equal or higher than 4 , then the order line is classified as $B_{H Q}$. Four carton boxes which are stored in a full layer on the pallet - can be more stable on a UL than just 1 carton box. If the ordered Quantity $(Q)$ is smaller than 4, then the order line $(r)$ is classified as $B_{L Q}$. Equations (5) and (6) show the example with the CartonBox class [11].

$$
\begin{gathered}
B_{L Q} \in P O P C \mid\left(r_{P C}=B\right) \wedge(Q<4) \\
B_{H Q} \in P O P C \mid\left(r_{P C}=B\right) \wedge(Q \geqslant 4) \\
\text { CartonBox }_{L Q} \in P O P C \mid\left(r_{P C}=\text { CartonBox }\right) \wedge(Q<4) \\
\text { CartonBox }_{H Q} \in P O P C \mid\left(r_{P C}=\text { CartonBox }\right) \wedge(Q \geqslant 4)
\end{gathered}
$$

\subsection{Classification of the special product and order parameter based classes}

We are defining Special POPCs (SPOPC) with considering previously picked units and their sequence (Eq. (7)). The picked items on the pallet form a physical structure which influence the choosing of the following items. For example: It is possible to pick 1 layer of small box, after 1 layer of small box, but the third layer small box would destabilize the unit load. So in this case it is forbidden to pick 1 layer small box after 2 layers small box (Eq. (8)) [11].

$$
S \in S P O P C \mid\left(P O P C_{t-1}=Y\right) \wedge\left(P O P C_{t-2}=Y\right)
$$

SmallBoxSmallBox $\in S P O P C \mid\left(P O P C_{t-1}=\right.$ SmallBox $) \wedge\left(P O P C_{t-2}=\right.$ SmallBox $)$

$t$ is the actual picking step, $t-1$ means the previously picked POPC, and $t-2$ is the last but one picked POPC. 


\subsection{Defining the PSFDM}

We are modeling the PSF based sequencing logic in a PSF based Decision Matrix (PSFDM). The predecessors (rows) are the elements of the Pallet Setup Class (PSC) set which is the union of POPC and SPOPC sets. The successors (columns) are the elements of the POPC set (Eqs. (9), (10)) [11].

$$
P S C=P O P C \cup S P O P C
$$

$$
P S F D M: P S C \times P O P C \mapsto\{0,1\}
$$

$$
P S F D M\left(P S C_{i}, P O P C_{j}\right)= \begin{cases}1 & \text {,if } P O P C_{j} \text { is able to be picked after } P S C_{i} \\ 0 & \text {,if } P O P C_{j} \text { is not able to be picked after } P S C_{i}\end{cases}
$$

The PSFDM values are 0 or 1 , depending on pallet setup possibilities. If it is possible to pick the examined item (one element of POPC) after the already picked units (PSC element), then the PSFDM value is 1 (true). Otherwise the picking is forbidden, so the PSFDM value is 0 (false) (Eq. (11)). Tables (1) and (2) illustrate an example PSFDM [11].

Table 1. Pallet Setup Feature based Decision Matrix (PSFDM).

\begin{tabular}{|c|c|c|c|c|c|}
\hline & $P O P C_{1}$ & $P O P C_{2}$ & $P O P C_{3}$ & $P O P C_{4}$ & $P O P C_{5}$ \\
\hline$P S C_{1}$ & 1 & 1 & 1 & 1 & 1 \\
\hline$P S C_{2}$ & 0 & 1 & 1 & 1 & 1 \\
\hline$P S C_{3}$ & 0 & 0 & 1 & 1 & 1 \\
\hline$P S C_{4}$ & 0 & 0 & 0 & 1 & 1 \\
\hline$P S C_{5}$ & 0 & 0 & 0 & 0 & 1 \\
\hline
\end{tabular}

Table 2. Pallet Setup Feature based Decision Matrix (PSFDM).

\begin{tabular}{|c|c|c|c|c|c|}
\hline & PlasticBin & CartonBox $_{H Q}$ & CartonBox $_{L Q}$ & SmallBox & Fragile \\
\hline PlasticBin & 1 & 1 & 1 & 1 & 1 \\
\hline CartonBox $H Q$ & 0 & 1 & 1 & 1 & 1 \\
\hline CartonBox $_{L Q}$ & 0 & 0 & 1 & 1 & 1 \\
\hline SmallBox & 0 & 0 & 0 & 1 & 1 \\
\hline Fragile & 0 & 0 & 0 & 0 & 1 \\
\hline
\end{tabular}




\section{Complexity of PSF based routing optimization}

We examined our problem to define the complexity of PSF based order picking routing optimization. In our case the examined warehouse can store its products in separated zones. It is a simple case where the warehouse has pallet setup based zones and the products behave on the same way within the zone. The order picking operator should visit the order picking zones in the right sequence, based on the PSFDM. Within the zone the picking sequence can be simple, based on any general heuristics as presented in Fig. 1.

Figure 1. Routing algorithms [2]

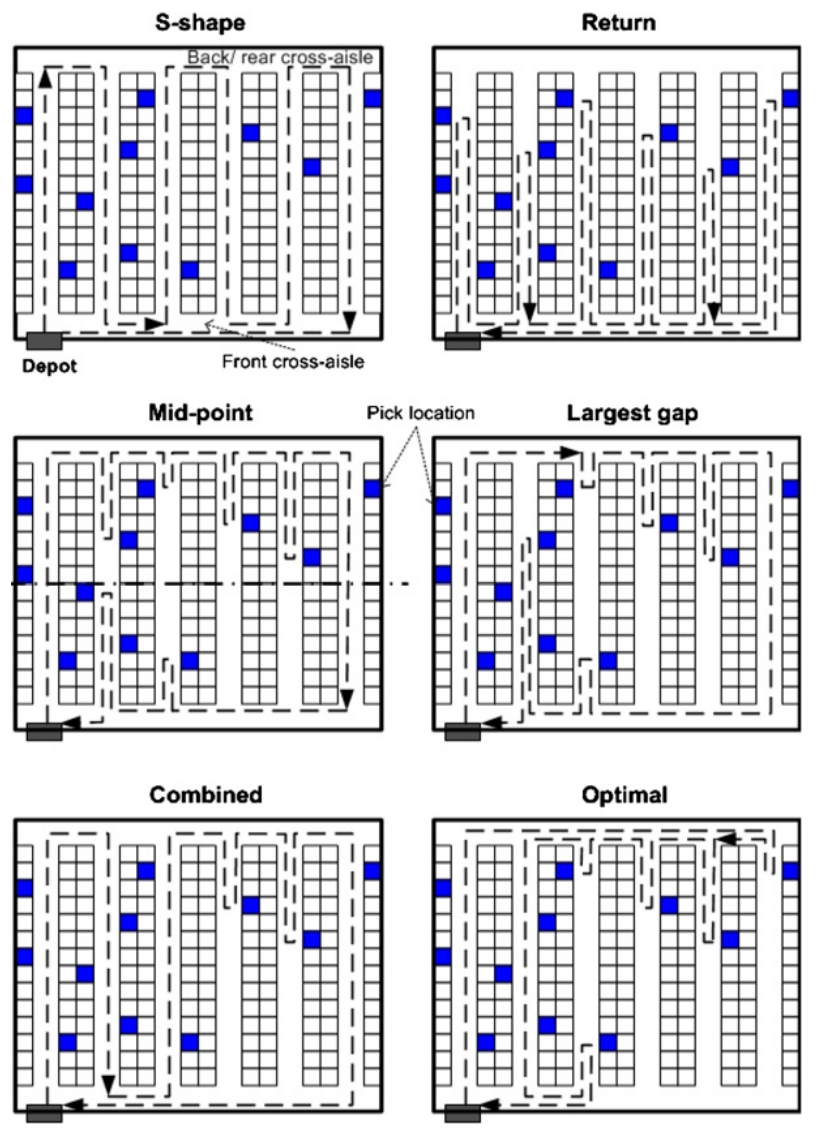

We determined a formula for calculating the possible number of zone sequencing combinations and examined its behavior when the length of order picking lists goes to 
infinity.

\subsection{Defining the number of combinations}

Each order picking list can contain every PSC any time and theoretically the sequence of the PSCs within the list is not important, while the rules of PSFDM are true. Therefore the possible cases of our problem is definable with the combination with repetition formula. Equation (12) shows the general formula of the combination with repetition, which results in the number of possible combinations [12].

$$
C_{k}^{n}=\left(\begin{array}{c}
n+k-1 \\
k
\end{array}\right)
$$

In our case $n$ means the number of Pallet Setup Classes and $k$ means the length of the order picking list. While the warehouses handle from $1(i=1)$ to $k(i=k)$ record long order picking lists, we have to summarize the possible combinations from 1 to $k$ (Eq. (13).

$$
\sum_{i=1}^{k}\left(\begin{array}{c}
n+i-1 \\
i
\end{array}\right)
$$

For example the order picking lists contain maximum 4 records $(k=4)$ and the number of defined PSCs are $4(n=4)$. When the length of order picking list equals $1(i=1)$, then the number of possible combinations equals $n$ (in our example it equals 4 ). When the order picking list is 2 records long $(i=2)$, then the number of possible combinations are $\left(\begin{array}{c}n+1 \\ 2\end{array}\right)$. We continue this sequence until $i=k$ and summarize the number of possible combinations to get the dimensions of the optimization.

Equation (13) is irreducible with the telescopic sum terms as shown in Eq. (14).

$$
\begin{gathered}
\sum_{i=1}^{k}\left(\begin{array}{c}
n+i-1 \\
i
\end{array}\right)=\sum_{i=1}^{k}\left(\left(\begin{array}{c}
n+i \\
i
\end{array}\right)-\left(\begin{array}{c}
n+i-1 \\
i-1
\end{array}\right)\right)= \\
\left(\left(\begin{array}{c}
n+1 \\
1
\end{array}\right)-\left(\begin{array}{c}
n+1-1 \\
1-1
\end{array}\right)\right)+\left(\left(\begin{array}{c}
n+2 \\
2
\end{array}\right)-\left(\begin{array}{c}
n+2-1 \\
2-1
\end{array}\right)\right)+ \\
\left(\left(\begin{array}{c}
n+3 \\
3
\end{array}\right)-\left(\begin{array}{c}
n+3-1 \\
3-1
\end{array}\right)\right)+\ldots+
\end{gathered}
$$




$$
\begin{gathered}
\left(\left(\begin{array}{c}
n+k-1 \\
k-1
\end{array}\right)-\left(\begin{array}{c}
n+k-1-1 \\
k-1-1
\end{array}\right)\right)+\left(\left(\begin{array}{c}
n+k \\
k
\end{array}\right)-\left(\begin{array}{c}
n+k-1 \\
k-1
\end{array}\right)\right)= \\
\left(\left(\begin{array}{c}
n+1 \\
1
\end{array}\right)-\left(\begin{array}{c}
n \\
0
\end{array}\right)\right)+\left(\left(\begin{array}{c}
n+2 \\
2
\end{array}\right)-\left(\begin{array}{c}
n+1 \\
1
\end{array}\right)\right)+\left(\left(\begin{array}{c}
n+3 \\
3
\end{array}\right)-\left(\begin{array}{c}
n+2 \\
2
\end{array}\right)\right)+\ldots+ \\
\left(\left(\begin{array}{c}
n+k-1 \\
k-1
\end{array}\right)-\left(\begin{array}{c}
n+k-2 \\
k-2
\end{array}\right)\right)+\left(\left(\begin{array}{c}
n+k \\
k
\end{array}\right)-\left(\begin{array}{c}
n+k-1 \\
k-1
\end{array}\right)\right)= \\
\left(\left(\begin{array}{c}
n+k \\
k
\end{array}\right)-\left(\begin{array}{c}
n \\
0
\end{array}\right)\right)=\left(\begin{array}{c}
n+k \\
k
\end{array}\right)-1
\end{gathered}
$$

The $\left(\begin{array}{c}n+k \\ k\end{array}\right)-1$ formula takes into consideration the PSF rules and defines the possible number of order picking zone sequencing combinations for order picking lists in case of standard triangle and symmetrical PSFDM. Our formula has some important facts:

- Many industrial cases are reducible to standard triangle and symmetrical PSFDM. However some special industrial cases can result in non-symmetrical non-standard triangle PSFDM, which might modify the real number of combinations.

- If we examine the inverse PSFDM, the number of possible combinations will be the same. It highlights, that our formula is not applicable into PSF based routing optimization without the PSFDM.

- $\left(\begin{array}{c}n+k \\ k\end{array}\right)-1=\left(\begin{array}{c}n+k \\ n\end{array}\right)-1$, because $\frac{(n+k) !}{n ! \cdot k !}-1=\frac{(n+k) !}{k ! \cdot n !}-1$

The goal of our formula is to highlight the complexity of our problem, in this case the specific cases and the inverse solutions are negligible. We can conclude, when we implement the PSFs into the order picking routing optimization, our algorithm should be able to handle unique (non-symmetrical and non-standard triangle) PSFDM with the exact picking rules.

\subsection{Proof of complexity}

When we implement a PSF based routing algorithm, the number of PSC-es $(n)$ will be parameter as an output of preceding system monitoring. Our algorithm will optimize the route of several order picking lists, whose length $(k)$ will be changing, depending on the customer orders. We examine the exponential growth of combinations with the following 
limits, where $n$ is an optional high integer number and $k$ goes to infinity. We examine our formula $\left(\begin{array}{c}n+k \\ k\end{array}\right)$ (where the -1 is a negligible term in case of limit value calculation) compared to the $e^{k}$ formula. Equations (15) and (16) highlight our hypothesis:

- If the defined limit goes to 0 then our formula's growing is faster than $e^{k}$ (Eq. (15)).

- If the defined limit goes to 1, then our formula's growing is the same as $e^{k}$ (Eq. (16)).

- If the defined limit goes to $\infty$, our formula's growing is slower than $e^{k}$. In this case, the described specific problem might not require any evolutionary optimization method (Eq. (17)).

$$
\begin{gathered}
\lim _{\substack{n \rightarrow N \\
k \rightarrow \infty}} \frac{e^{k}}{\left(\begin{array}{c}
n+k \\
n
\end{array}\right)} \stackrel{?}{=} 0 \\
\lim _{\substack{n \rightarrow N \\
k \rightarrow \infty}} \frac{e^{k}}{\left(\begin{array}{c}
n+k \\
n
\end{array}\right)} \stackrel{?}{=} 1 \\
\lim _{\substack{n \rightarrow N \\
k \rightarrow \infty}} \frac{e^{k}}{\left(\begin{array}{c}
n+k \\
n
\end{array}\right)} \stackrel{?}{=} \infty \\
\lim _{\substack{n \rightarrow N \\
k \rightarrow \infty}} \frac{e^{k}}{\left(\begin{array}{c}
N+k \\
N
\end{array}\right)}=\lim _{\substack{n \rightarrow N \\
k \rightarrow \infty}} \frac{e^{k}}{(N+k) !} \\
\lim _{\substack{n \rightarrow N \\
k \rightarrow \infty}} \frac{\lim _{n \rightarrow N} \frac{e^{k} \cdot N ! \cdot k !}{(N+N+K) !}}{(N+k) \cdot(N+k-1) \cdot(N+k-2) \cdot \ldots \cdot(N+1) \cdot \mathbf{N} \cdot \ldots \cdot \mathbf{3} \cdot \mathbf{2} \cdot \mathbf{1}}= \\
\lim _{\substack{n \rightarrow N \\
k \rightarrow \infty}} \frac{e^{k} \cdot \mathbf{N} ! \cdot k !}{(N+k) \cdot(N+k-1) \cdot(N+k-2) \cdot \ldots \cdot(N+3) \cdot(N+2) \cdot(N+1)}=\infty
\end{gathered}
$$

Equation (18) proves, that our formula goes to $\infty$, because each $\frac{k e}{(N+k)}, \frac{((k-1) \cdot e)}{(N+(k-1))}$, $\frac{((k-2) \cdot e)}{(N+(k-2))}, \ldots$ quotient goes to $e$ and there are $k$ quotients, so the result is $\infty$. 
Since $\left(\begin{array}{c}n+k \\ k\end{array}\right)-1=\left(\begin{array}{c}n+k \\ n\end{array}\right)-1$, we will get the same result if $n$ goes to infinity and $k$ is an optional high integer number.

The result means, that $\left(\begin{array}{c}n+k \\ k\end{array}\right)-1$ has less than exponential growth. Thus, evolutionary optimization method is not necessary for the described simple cases.

\section{Conclusion}

Pallet Setup Feature is one of the features that can be important for a warehouse. It depends on various item properties, order parameters, and order picking systems, but not relevant at every warehouse. If it is, its examination is essential during an OPP development. In the paper we described our methodology for modeling pallet setup rules. By classifying the products based on product and order parameters we can define a Pallet Setup Feature based Decision Matrix (PSFDM), which will be the basis of PSF based routing algorithms. These algorithms define a possible picking sequence to avoid the product damages and increase the OPP effectiveness. Routing optimization is essential at every warehouse, because it is the most time consuming movement of the order picking but its required complexity could be different warehouse by warehouse [2]. To find out how complex optimization do we need, we have to examine the complexity of the given case.

Through a simple case when the products of the defined pallet setup classes are stored in separated zones, we examined the complexity of the pallet setup features based routing. We determined a formula for calculating the possible number of zone sequencing combinations and examined its behavior when the length of order picking lists goes to infinity. We can conclude, when the products are stored in separated PSC zones, the order picking operators visit the zones in PSFDM based sequence and collect the items with general routing algorithms (Fig. (1)) within the zone, then non-evolutionary algorithms can handle the problem and support the operator with right picking sequence.

\section{References}

[1] T. Le-Duc and S. W. J. Lamberts, Design and Control of Efficient Order Picking Processes. Rotterdam, Netherlands: Erasmus University Rotterdam Press, 2005.

[2] R. de Koster, T. Le-Duc, and K. J. Roodbergen, "Design and control of warehouse order picking: A literature review," European Journal of Operational Research, vol. 182, pp. 481-501, 2007.

[3] J. Ashayeri and M. Goetschalckx, "Classification and design of order picking," Logistics Inf Manage, vol. 2, no. 2, pp. 99--106, 1989.

[4] K. L. Choy, H. Y. Lam, L. Canhong, and C. K. H. Lee, "A hybrid decision support system for storage location assignment in the fast-fashion industry," in Technology 
Management in the IT-Driven Services (PICMET), 2013 Proceedings of PICMET '13, (San Jose, California, USA), pp. 468—473, August 2013.

[5] R. Alvarez-Valdes, F. Parreño, and J. M. Tamarit, "A tabu search algorithm for the pallet loading problem," OR Spectrum, vol. 27, pp. 43-61, 2005.

[6] K. Moeller, "Increasing warehouse order picking performance by sequence optimization," Procedia Social and Behavioral Sciences, vol. 20, pp. 177-185, 2011.

[7] F. T. Chan and H. Chan, "Improving the productivity of order picking of a manualpick and multi-level rack distribution warehouse through the implementation of class-based storage," Expert Systems with Applications, vol. 38, pp. 2686-2700, 2011.

[8] J. Y. Shiau and M. C. Lee, "A warehouse management system with sequential picking for multi-container deliveries," Computers and Industrial Engineering, vol. 58, pp. 382-392, 2010.

[9] T. Bartók and C. Imreh, "Pickup and delivery vehicle routing with multidimensional loading constraints," Acta Cybernetica, vol. 20, no. 1, pp. 17-33, 2011.

[10] T. Bódis, K. Udvardy, and J. Botzheim, "Interactive training and modeling environment for considering pallet setup features in storage location assignment of order picking zone," in Proc. of The Mecatronics-2014-Tokyo, (Tokyo, Japan), pp. 64-94, November 2014.

[11] T. Bódis and J. Botzheim, "Modelling order picking sequencing variations of pallet setup clusters," in Proc. of The International Conference on Logistics and Sustainable Transport 2015, (Celje, Slovenia), pp. 86-94, June 2015.

[12] M. Bóna, A Walk Through Combinatorics: An Introduction to Enumeration and Graph Theory - Third Edition. Singapore: World Scientific Publishing Co. Pte. Ltd., 2011. 